\title{
Incidence of Adverse Drug Events in Secondary Hospital at Cabo Verde Identified Using Trigger Tools
}

\author{
Carla Djamila Reis*, Calida Etzana Veiga and Jailson Jesus Martins \\ Agência de Supervisão e Supervisão de Produtos Farmacêuticos e Alimentares (ARFA), Praia, Cape Verde
}

\begin{abstract}
Background: Adverse drug events (ADE) are a major health and economic problem. There is no information regarding incidence of $A D E$ in Cabo Verde and trigger tools are an efficient active data collection method.

Objective: To develop the first PV study in Cabo Verde and characterize ADE in a hospital.

Method: This is a retrospective study of chart review using the Global Trigger Tool (GTT) developed by the Institute for Healthcare Improvement $(\mathrm{IHI})$. The simple random sample of medical records for patients discharged from October 2012 to March 2013 and from October 2013 to March 2014. Hospitalization for less than $48 \mathrm{~h}$, time spent in intensive care unit and medical records with no drug administration records were excluded.

Results: A total of 383 medical records data were collected. The average age of patients was 37 and 40 years $62.6 \%$ and $59.8 \%$ of the total were females. A total 287 triggers were identified, 184 and 103 triggers for first and second period, respectively. For the first period, a total of $105 \mathrm{ADE}$ were identified in 54 patients with the incidence of $28.4 \%$ patient presenting $\mathrm{ADE}$ and a rate of $54 \%$ ADEs per 100 patients, in the second period, a total of $77 \mathrm{ADE}$ were identified in 38 patients with the incidence of $19.6 \%$ and a rate of $38 \%$ ADE per 100 patients. Drugs more often associated with an $\mathrm{ADE}$ were ceftriaxone, furosemide, metronidazole and ranitidine.
\end{abstract}

Conclusion: The trigger tool had a good performance detecting ADE. About $1 / 4$ of the hospitalized patients in hospital showed adverse events. Further studies are needed using prospective method and extended period.

Keywords: Pharmacovigilance; Cabo Verde; Safety monitoring; Adverse drug event monitoring; Trigger tool; Hospital pharmacovigilance

\section{Background}

\section{The relevance and study methodologies in hospital}

Adverse drug events (ADE) are frequent, a leading cause of morbidity, mortality and furthermore, costly [1]. It is estimated that the overall incidence of ADE, including both those that cause hospitalization and those that occur during hospitalization, is $15.1 \%$, and in this range $6.7 \%$ are severe [2]. Another systematic review of 29 studies found between 1.7 and 51.8 events per 100 hospitalizations [3].

In the sub-Saharan African region where Cabo Verde is located, there are few pharmacovigilance (PV) studies. Uganda, one of the four countries assessed by WHO as having installed pharmacovigilance capacity [4], identified that $4.5 \%$ of patients were hospitalized with $\mathrm{ADE}, 1.6 \%$ were hospitalized due to ADE and that ADE occurs during hospitalization in $49.5 \%$ of patients. The ADE were found to be an important morbidity factor for both community and hospital and is associated with the most widely used drugs [5].

Different approaches and methodologies have been used to characterize the incidence of ADE. The clinical studies, the spontaneous reporting (NE), the algorithms for database search and the chart review proven to have disadvantages such as being expensive, insensitive or largely ineffective [6,7]. The use of triggers during review of medical records, appears as an alternative to overcome this disadvantages emerging as the premier measurement strategy for patient safety $[8,9]$.

This method consists in a list of triggers previously tested, including medicines, laboratory findings and information on assistance to the patient and clinical outcome that act as clues to identify $\operatorname{ADE}[10,11]$.

In Cabo Verde, there are no studies or data on the incidence of adverse reactions. In the context of a research project to develop a proposed model for the implementation of a pharmacovigilance system adapted to the national reality it was deemed important to have national data on the incidence of ADE. This study was undertaken in a hospital with validated triggers tools with the objective of testing the use of the methodology and characterize the ADE identified.

\section{Method}

\section{Study settings and design}

The study was done at a secondary care hospital in Santiago, Cabo Verde. This hospital, with 90 beds, covers six municipalities whose population, about 112000 inhabitants, represent $48.8 \%$ of the population of the island of Santiago and $27.2 \%$ of the country.

The study plan was submitted to the Ethics Committee for Health Research at the Ministry of Health and then approved by the hospital board. A multidisciplinary team was appointed to discuss procedures and to follow up the study.

This retrospective descriptive study had as inclusion criteria a minimum hospitalization period of $48 \mathrm{~h}$, records with drug administration, complete discharge summaries and coding. Cases of

*Corresponding author: Carla Djamila Reis, Master in Pharmaceutical Regulation, Agência de Regulação e Supervisão, de Produtos Farmacêuticos e Alimentares (ARFA), Praia, Cape Verde, Tel: 23826264 10; E-mail: djamila.reis@arfa.gov.cv

Received October 26, 2015; Accepted November 13, 2015; Published November 18, 2015

Citation: Reis CD, Veiga CE, Martins JJ (2015) Incidence of Adverse Drug Events in Secondary Hospital at Cabo Verde Identified Using Trigger Tools. J Pharmacovigil 3 183. doi:10.4172/2329-6887.1000183

Copyright: @ 2015 Reis CD, et al. This is an open-access article distributed under the terms of the Creative Commons Attribution License, which permits unrestricted use, distribution, and reproduction in any medium, provided the original author and source are credited. 
Citation: Reis CD, Veiga CE, Martins JJ (2015) Incidence of Adverse Drug Events in Secondary Hospital at Cabo Verde Identified Using Trigger Tools. J Pharmacovigil 3: 183. doi:10.4172/2329-6887.1000183

emergency ward and intensive care were excluded.

The medical records were randomly selected based on the total number of hospitalization by simple sampling (SAS) with a $5 \%$ error and a $9 \%$ increase from the number calculated in order to allow exclusion of processes incomplete or impossible to assess. 383 medical records for a period of analysis corresponding to 12 months were selected in two phases based on the date of admission. The first phase included 190 processes for the period October 2012 to March 2013. A subsequent random selection of other 193 medical records was made for the period of October 2013 to March 2014.

A review of the processes was performed using the Global Trigger Tool (GTT), a tool developed by the Institute for Healthcare Improvement (IHI), consisting of a set of triggers used to identify potential adverse events [11] as adapted in published studies on the implementation and performance of those triggers (Table 1) $[12,13]$.

\section{Adverse event definition}

$\mathrm{ADE}$ are the occurrence of any harm to the patient potentially related to medical intervention with the use of medication, resulting in a temporary or permanent physical or psychological disturbance in the body or in its structure. The definition includes prescribing, dispensing and administrating errors and adverse reactions [10]. Events existing upon hospital admission were also registered.

\section{Data collection}

Data collection and review of medical records were planned and executed by a team of a pharmacist responsible for the study, a graduate in biomedicine, a technical statistical responsible for the design of the database and the hospital technician responsible for the storage of files.

A specific database for this study has been developed in Microsoft Office Access 2010 with the help of Visual Basic for Applications programming language for data entry. The forms have fields that incorporate structured and customized validations in order to avoid errors in typing process.

The data collected refer to the demographic, social, health and hospitalization: gender, age, occupation, education, date and inpatient reason, diagnosis, concomitant diseases, discharge summary, medication administered and whether or not one or more of the triggers were identified. When triggers are identified the reviewer has to further analyze if there is an ADE. In case of existing ADE the assessment is made in terms of expectedness, seriousness according to WHO categories, and severity according to National Coordinating Council for Medication Error Reporting and Prevention Index (NCC MERP) and causality, for each adverse event and each drug, using the Naranjo algorithm, except for drugs administered to treat the event.

\section{Variables of the study and statistical analysis}

$\mathrm{ADE}$ occurring during hospitalization was classified as the result of the study. The variables evaluated in this study are: age, gender, type of admission (outpatient, emergency, transfer), discharge situation (transfer, clinic, death), days of hospitalization, number of medicines used, classified according to the anatomic therapeutic classification (ATC) and the medical diagnosis classified according to International classification of diseases ICD-10.

Data analyzes and data processing was performed, using SPSS version 20.

\section{Results}

A total of 383 medical records data were collected. All results will be presented for the first period with 190 medical records from October 2012 to March 2013 and for the second period with 193 medical records from October 2013 to March 2014.

\section{Characterization of population}

In general, the samples are comparable as regards to the demographic characteristics. The average age of patients was 37 and 40

\begin{tabular}{|c|c|c|c|}
\hline & \multicolumn{2}{|c|}{ Trigger } & Potential ADE \\
\hline \multirow{9}{*}{ 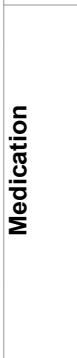 } & T1 & Anti-Allergic & Hypersensitivity Reaction \\
\hline & $\mathrm{T} 2$ & Coagulant & Overdose of warfarin \\
\hline & T3 & Benzodiazepine Antagonist & Sedation for benzodiazepines \\
\hline & T4 & Anti-emetic & Nausea /vomiting \\
\hline & T5 & Opioid Antagonist & Narcotic drugs overdose \\
\hline & T6 & Antidiarrheal & Gastrointestinal Effects \\
\hline & T7 & Ion Exchange Resin & Hyperkalemia \\
\hline & T8 & Digoxin & \\
\hline & T9 & Abrupt medication stop & \\
\hline \multirow{7}{*}{ 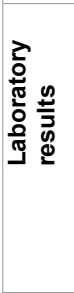 } & T10 & Partial thromboplastin time greater than 100 Seconds & Excessive anticoagulation with heparin \\
\hline & T11 & International Normalized Ratio (INR) greater than 6 & Excessive anticoagulation with warfarin \\
\hline & T12 & White blood cell (WBC) count less than $3000 \times 106 / \mu l$ & Neutropenia \\
\hline & T13 & Glucose less than $50 \mathrm{mg} / \mathrm{dl}$ & Hypoglycemia associated with insulin use \\
\hline & T14 & Increase in serum creatinine & Renal failure \\
\hline & T15 & Clostridium difficile positive stool & Exposure to antibiotics \\
\hline & T16 & Platelet count less than 50.000 & \\
\hline \multirow{5}{*}{ 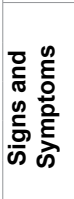 } & T17 & Over-sedation, lethargy, fall & ADE \\
\hline & T18 & Rash & ADE \\
\hline & T19 & Transfer to higher level of care & ADE \\
\hline & T20 & Medical description & ADE \\
\hline & T21 & Nurse description & ADE \\
\hline
\end{tabular}

Table 1: List of triggers to identify on medical records. 
years, $62.6 \%$ and $59.8 \%$ of the total were females, the education level lied mostly between the basic (15.3\% and 16.5\%) and high school $(16.3 \%$ and $21.6 \%)$ and most patients were single (40\% and 38.1\%) for each period, respectively. The hospitalization time is equal to or less than 10 days for $76.7 \%$ and $68.1 \%$ of cases for each period, respectively. The number of drug administered ranges from 1 to 19 and 1 to 20 with an average of 5 and 4 in each period.

\section{Characterization of triggers}

Of the 383 medical records reviewed, a total 287 triggers were identified, 184 and 103 triggers for $1^{\text {st }}$ and $2^{\text {nd }}$ period, respectively. During the first period most of medical records had 1 to 4 triggers (55.8\%) and for the second period it was $27.5 \%$. The proportions of medical records with 5 or more triggers were $1.1 \%$ and $1.5 \%$.

The analysis of total triggers identified in the medical records reviewed during the study period identified the description of the nurse and the physician as the two most identified that account for about half of all identified triggers (Figure 1) (31.7\% and 19.2\%, respectively).

Analysing and comparing the specific triggers identified (Figure 2); the most identified trigger is the description of the nurse for both periods (33\% and $29 \%)$.

\section{Incidence of ADE}

The incidences of ADE were calculated for each period dividing the number of patients with at least one adverse event by the total number of patients.

For the first period, a total of $105 \mathrm{ADE}$ were identified in 54 patients with the incidence of $28.4 \%$ patient presenting ADE and a rate of $54 \%$ ADE per 100 patients. In the second period, a total of $77 \mathrm{ADE}$ were identified in 38 patients with the incidence of $19.6 \%$ and a rate of $38 \%$ ADE per 100 patients.

In both periods, the majority of $\mathrm{ADE}$ was non-serious, as per WHO definition (52.4\% and 63.1\%). The most serious ADE identified were "persistent or significant incapacity" (19.4\%) during the first period and "life-threatening" during the second period.

The classification of severity made in accordance with National Coordinating Council for Medication Error Reporting and Prevention (NCC MERP) identifies $76.5 \%$ and $89.2 \%$ of severe ADE in the $1^{\text {st }}$ and $2^{\text {nd }}$ period, respectively. The most identified categories were "Temporary harm to the patient and required intervention" $(35.0 \%$ and $77.7 \%$ for first and second period, respectively).

The majority of ADE identified was expected according to the approved summary of product characteristics and had similar distribution between the two periods ( $4.2 \%$ and $47.6 \%)$.

The causality assessment using the Naranjo algorithm was made for all ADE and for all the drugs used by each patient, except for those used in SOS and to correct or treat the event itself. In both periods the majority of the degree of association after causality assessment was possible ( $83.3 \%$ and $87.1 \%$ ) followed by doubtful (16\% and $11.7 \%$ ).

The distribution of ADE according SOC is reflected in Figure 3 where highlights the gastrointestinal disorders. In both periods, the most commonly implicated drugs in ADE were ceftriaxone, metronidazole, ranitidine and furosemide.

\section{Discussion}

We have examined data in 384 medical records in a hospital

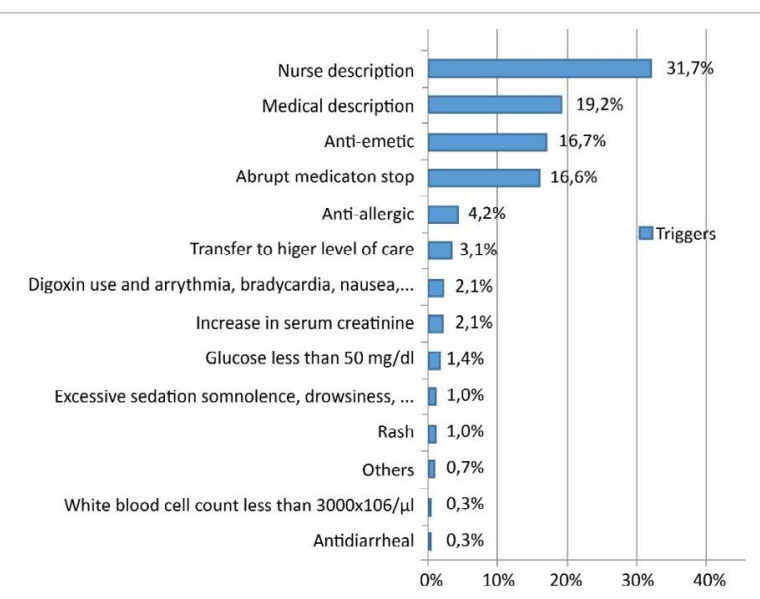

Figure 1: Total of triggers identified on total 383 medical records.

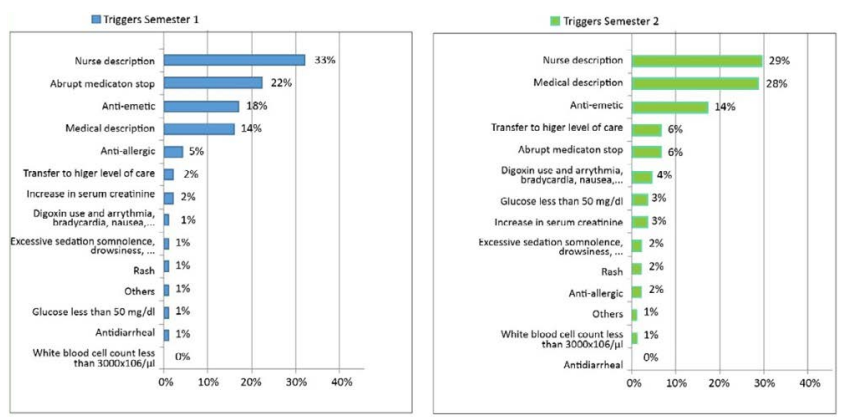

Figure 2: Comparison of triggers identified in the study population in the 2 periods.

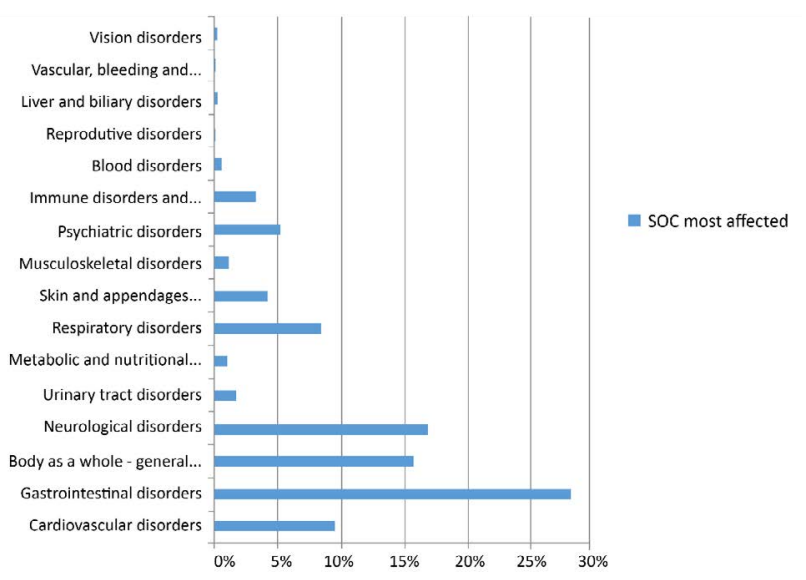

Figure 3: Most affected SOC.

using a trigger tool to identify $\mathrm{ADE}$ using a team of two healthcare professionals.

The results related to the demographic characteristics of the population in both periods of hospitalization, from October 2012 to March 2013 and from October 2013 to March 2014, can be considered roughly comparable as per the number, sex distribution, age, and level of schooling, marital information, and number of days of hospitalization, average number of medicines per patient and discharge situation.

The analysis of total triggers identified during the study period 
brings out the role of the health care professional considering that the description of the nurse and the physician are the two most identified and account for about half of all identified triggers.

About $28.4 \%$ and $19.6 \%$ of inpatients have experienced ADEs, during the first and second period respectively. The study conducted in the United States with six community hospitals, estimated a rate of 15.0 ADEs per 100 patients [7]. In other study, researchers encountered in a Brazilian hospital a rate of 26.6 ADEs per 100 patients [12]. Some characteristics of the study population, the hospital's profile and previous existence of pharmacovigilance system may explain the differences.

As regards to degree of harm, using National Coordinating Council for Medication Error Reporting and Prevention Index, most of the events resulted in temporary patient harm that required some intervention and a similar proportion of events (79.9\%) in the Brazilian study [10]. Comparing with the same study similar results were found concerning most involved SOC (gastrointestinal) and medicines (ranitidine). The advantages of this study was that we found that the trigger tool can be efficiently applied with a modest amount of training and that it allowed identifying ADE and have national information for the first time and that will be useful to assess the effectiveness of interventions to improve patient safety over time. Other strength was a randomly selected sample calculated to represent a hospital that attends almost $1 / 3$ of the country population. As concluded in previous study [7], it suggests that this method can be used to determine national rates of adverse events. This information has crucial importance in a context of establishment of the pharmacovigilance system, of including hospitals as a key element and for promotion of notification by health care professionals.

The proportion of total and serious ADE identified seems to reinforce the relevance of this methodology that would be of major benefit using prospective study design that could address the preventable ADE. As for other limitations of this study the lack of automated systems in the hospital was identified because the aid of screening criteria used as filters for preliminary analysis of information in medical records would provide a more objective and viable alternative to traditional patient chart reviews. This disadvantage was already identified in previous study [3] and had a direct impact in the time used to analyze medical records that was approximately 3 times the 20 minutes described for the method. Therefore, the method was found important but not suitable as routine method to support a pharmacovigilance system. Other limitation, also identified in the same study, was the difficulty to distinguish an ADE form the underlying disease.

There is a need for further studies to identify if the causality assessment results are related to the filling system, information systems and electronic database that could allow harmonizing and having more complete medical records.

\section{Conclusion}

This report represents the first pharmacovigilance study at a hospital in Cabo Verde and describes the application of a practical method for quantifying the occurrence of ADE in hospitalized patients. Safe and cost-effective use of medicines can be promoted by such studies.

This trigger tool, even without the use of electronic databases, has proven possible to be used to detect ADE and may realistically be expected to be useful in improving clinical processes.

In the context of establishing a pharmacovigilance system adapted to the country's reality, this method does not adapt to a routine identification but is very important for further studies and AEs characterization. Implementing this relatively low cost program in a low budget hospital allowed to have first national data and is thought to increases interest, knowledge and became possible a generalized application of the method to consistently support a pharmacovigilance system that is being established.

Recommendations to retain from this study is the need to establish procedures and support tools that promote complete and harmonized information, legible and possible to extract for analysis which is to say, file and information systems. It would be possible to take advantage of this kind of study and even promote the adoption of a list of triggers in hospitals that reinforce the spontaneous reporting system to be established. The merge of both methods would promote an increasingly safe and cost-effective use of medicines in Cabo Verde.

\section{Acknowledgement}

To the Hospital Director, Clinical Director and Statistic technician, Dr Dalia Monteiro, Dr Khadija Carvalho and Ms Cecilia Joel, respectively.

To Agência de Regulação e Supervisão dos Produtos Farmacêuticos e Alimentares (ARFA)

To the Direction of Pharmaceutical Regulation, Dr. Eduardo Tavares.

\section{References}

1. Miguel A, Azevedo LF, Araújo M, Pereira AC (2012) Frequency of adverse drug reactions in hospitalized patients: A systematic review and meta-analysis. Pharmacoepidemiol Drug Saf 21: 1139-1154.

2. Lazarou J, Pomeranz BH, Corey PN (1998) Incidence of adverse drug reactions in hospitalized patients: a meta-analysis of prospective studies. JAMA 279 : 1200-1205.

3. Cano FG, Rozenfeld S (2009) Adverse drug events in hospitals: a systematic review. Cad Saúde Pública 25: S360-S372.

4. Strengthening Pharmaceutical Systems (SPS) Program (2011) Safety of Medicines in Sub- Saharan Africa: Assessment of Pharmacovigilance Systems and their Performance.

5. Tumwikirize WA, Ogwal-Okeng JW, Vernby A, Anokbonggo WW, Gustafsson LL, et al. (2011) Adverse drug reactions in patients admitted on Interna Medicine wards in a district and Regional Hospital in Uganda. Afr Health Sci 11: 72-78.

6. de Wet C, Bowie P (2009) The preliminary development and testing of a global trigger tool to detect error and patient harm in primary-care records. Postgrad Med J 85: 176-180

7. Sharek PJ, Parry G, Goldmann D, Bones K, Hackbarth A, et al. (2011) Performance characteristics of a methodology to quantify adverse events over time in hospitalized patients. Health Serv Res 46: 654-678.

8. Sharek PJ (2012) The Emergence of the Trigger Tool as the Premie Measurement Strategy for Patient Safety. AHRQ WebM\&M 2012: 120.

9. Naessens JM, O’Byrne TJ, Johnson MG, Vansuch MB, McGlone CM, et al (2010) Measuring hospital adverse events: assessing inter-rater reliability and trigger performance of the Global Trigger Tool. Int J Qual Health Care 22: 266274

10. Rozich J, Haraden C, Resar R, et al. (2003) Adverse drug event trigger tool: a practical methodology for measuring medication related harm. Qual Saf Health Care 12: 194-200.

11. Griffin FA, Resar RK (2009) IHI Global Trigger Tool for Measuring Adverse Events. ( $2^{\text {nd }}$ Edition) $\mathrm{IHI}$ Innovation Series white paper, MA: Institute for Healthcare Improvement, Cambridge.

12. Giordani F, Rozenfeld S, Oliveira DFMD, Versa GLGDS, Terencio JS, et al. (2012) Vigilância de eventos adversos a medicamentos em hospitais: Aplicação e desempenho de rastreadores. Revista Brasileira de Epidemiologia 15: $455-467$

13. Roque KE, Melo ECP (2010) Adaptação dos critérios de avaliação de eventos adversos a medicamentos para uso em um hospital público no Estado do Rio de Janeiro. Revista Brasileira de Epidemiologia 13: 607-619. 